\title{
Representations and Character Recognition: An Issue for Action in Perception
}

\author{
Fiona M Richardson ${ }^{1,2}$ (F.1.Richardson@herts.ac.uk) \\ Sue Anthony ${ }^{2}$ (S.H.1.Anthony@herts.ac.uk) \\ John Done ${ }^{2}$ (D.J.Done@herts.ac.uk) \\ Lorna Peters ${ }^{1}$ (L.Peters@herts.ac.uk) \\ Neil Davey' (N.Davey@herts.ac.uk) \\ Departments of Computer Science ${ }^{1}$ and Psychology ${ }^{2}$, University of Hertfordshire, College Lane, Hatfield, \\ AL10 9AB, United Kingdom.
}

\begin{abstract}
Considering the growing interactive relationship of perception and action, building on Freyd's (1983a, b) work, a series of experiments was conducted to investigate the implied role of action knowledge in the predominantly perceptual task of character recognition. The outcome of this experimental work highlights the issue that action may interfere with learning, through the generation of conflicting representations. In addition, the results draw attention to complex issues involved in investigating the role of action within perception
\end{abstract}

\section{Introduction}

Traditionally, perception and action have been viewed as two separate systems independent of each other (Sternberg, 1969), with perception directing and guiding action (Ward, 2002). This flow of information from perception to action during processing is considered to be unidirectional. Therefore, within this framework, the converse case of action influencing and informing perception can only take place on completion of processing.

However, in recent psychological literature the relationship between perception and action has grown to the point where they are considered to be inseparable partners in cognition (Glenberg, 1997; Barsaolu,1999), making the bi-directional interaction between perception and action during processing almost theoretically essential. This view is growing in popularity, and the framework has even extended to the issue of communication between the two systems (Prinz, 1997).

The search for empirical evidence in support of a more interactive perception and action system has spread from Neurology to Experimental Psychology. In Neurology, evidence of visuomotor neurons, which fire whilst specific actions are observed has been provided as a testament to a more integrated perception-action system (Rizzolatti, 1994; Fadiga, Fogassi, Gallese \& Rizzolatti, 2000). In Experimental Psychology, Freyd (1983a) demonstrated that motion is represented when static stimuli are viewed. Also, Van den Bergh, Vrana \& Eelen (1990), demonstrated that, in a simple forcedchoice task, in which typists and non-typists were required to select a preferred letter pair, typists showed a strong preference for letter-pair combinations typed with a different finger, in comparison to letter-pairs typed with the same finger. Non-typists showed no preference. Evidence such as this is taken to strongly suggest the inclusion of motor information in the representations used during perceptually based tasks.

Earlier work, such as that by Freyd (1983b) is suggestive of the use of motor representations to support the predominantly perceptual task of character recognition. In this experiment participants viewed characters being drawn and were required to learn the corresponding digit for each character. After learning, participants carried out a forced-choice recognition task, in which undistorted and distorted characters were displayed. The results of this experiment showed that participants recognised characters distorted in a manner consistent with the drawing method observed during learning, faster than characters distorted in a manner inconsistent with the drawing method learnt. The reaction times for consistent distortions were very similar to those for undistorted characters. Freyd concluded that (a) knowledge of how letters are formed is used in the character recognition process, and (b) that participants were able to infer the dynamic pattern of motor movements used to produce the characters. This work implies the use of some form of motor-based production information being responsible for the dynamic effect found by Freyd. However, the evidence is not conclusive: the representations formed during learning were based upon the visual experience of watching the characters being drawn on-screen, and so the motor component cannot be directly implicated as a contributor to the formation of the representations during learning, that were subsequently used in recognition. Therefore, the origin of the information responsible for the effect found, being either motoric in 
origin, or some form of dynamic visual information, remains unresolved.

In order to further explore the effect found by Freyd, an experimental paradigm was developed in which, during learning, participants acquired motor experience of drawing the characters as opposed to simply watching them being drawn. It was expected that by introducing this motor experience, the effect found by Freyd (1983b) would be enhanced, as participants would be drawing upon existing motor representations rather than inferring them. This paper describes a sequence of three studies: the first two examine the influence of the introduction of motoric experience during learning in character recognition. As a result of the findings of these experiments, the original effect obtained by Freyd is examined in further detail.

\section{Experiment One}

This experiment was designed in terms of materials and procedure in the spirit of the original Freyd (1983b) task. The main alteration, however, was that for this experiment learning was centered upon the motor experience of learning to draw the characters, as opposed to participants simply viewing the production of the characters. It was expected that the motor representations formed during learning would contribute to the recognition of the characters, thus enhancing the original effect found by Freyd.

\section{Method}

Participants A total of 16 right-handed undergraduate students, 8 males and 8 females from the University of Hertfordshire took part in this experiment.

Materials and apparatus consisted of a Learning Set of 5 undistorted characters as shown in Figure 1. These characters could be drawn according to two drawing methods (A or B), differing in the direction in which the strokes used to produce the character were drawn. The completed undistorted character was visually the same for both drawing methods.

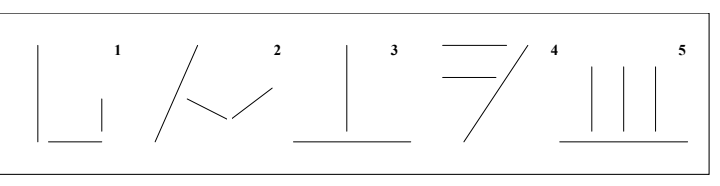

Figure 1: shows the learning set of five undistorted characters which participants were required to draw to one of the predetermined drawing methods. It should be noted that character two is taken from Babcock and

Freyd (1988), as this character was shown to produce strong dynamic effects in a related task.

There was also a Test Set consisting of 40 characters. 15 of these characters were distorted in a manner consistent with drawing method $\mathrm{A}$, and 15 distorted in a manner consistent with drawing method B. The 10 remaining characters were the Learning Set of undistorted characters, repeated. An example of the different distortion categories derived from real distortions of the Learning Set can be seen in Figure 2 . These stimuli were delivered using a 486 PC all phases.

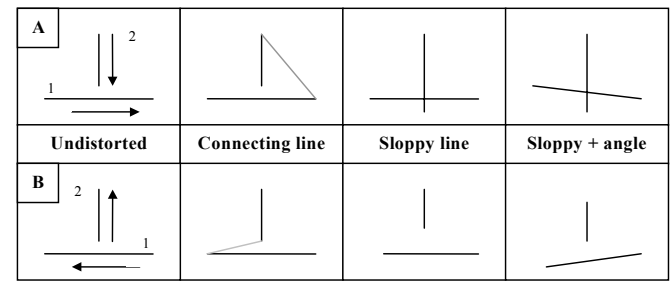

Figure 2: shows the two different drawing methods applied to a single character, and an example of a set of distortions for drawing method A (at top) and B (at bottom). It can be seen that the distortions produced between the two drawing methods are quite different in appearance. Distortions were derived from actual distortions collected in pilot work, which involved collecting characters drawn by participants under a time constraint.

Procedure The experiment was conducted in three phases. (i) the introductory phase, (ii) the learning phase and (iii) the test phase. During the (i) introductory phase, participants watched each character being drawn upon the computer screen individually, in numeric order, according to a selected drawing method. During this time the corresponding digit for each character was displayed in the top-right corner of the screen. The selection of the drawing method at the introductory phase was counter-balanced and was then fixed for the following learning phase. In the (ii) learning phase participants learnt to draw each character according to the selected drawing method, and associate each character with its corresponding digit. In order to reduce the number of production errors and distortions during learning, participants first traced over the character using a mouse as it was being drawn using the computer. Upon completion of two successful traces, participants then drew the character from memory. Any errors made during this learning process were logged and deleted from the screen, participants were given a correctional hint and required to repeat the trace or drawing attempt for that character. Upon completion of all successful tracing and drawing attempts participants were required to input the corresponding digit for the character in order to continue. The learning phase was completed when two cycles of the learning process for all characters was completed. In the (iii) test phase participants were presented with a static character randomly selected 
from the Test Set, and required to choose one of two digits displayed in the top corner of each side of the screen. Participants were informed that there was only one correct response and that some characters may be distorted, but that it should not interfere with their identification. During the test phase, the entire test set was presented a total of three times. Following this participants were tested in their ability to recall the characters learnt and label each character according to its corresponding digit. This was done to exclude from further analysis the data of participants who were unable to correctly identify all undistorted characters.

Results Reaction time data recorded during testing were analysed. A two-way mixed ANOVA was conducted on the data. Drawing method learnt (A or B) was a between-subjects factor, and type of distortion (three levels: undistorted, consistent and inconsistent) was a within-subjects factor. A significant main effect of type of distortion [F $(2,28)=19.127, p<0.001]$ was found. As there was no main effect of drawing method $[\mathrm{F}(2,28)=0.034 \mathrm{p}>0.05]$ or interaction between method and type $[\mathrm{F}(2,28)=0.954, \mathrm{p}>0.05]$ data were collapsed across drawing methods for subsequent analysis. A further one-way ANOVA (within-subjects) was conducted upon the collapsed data. A significant effect of type of distortion was found $[F(2,30)=$ $16.075, \mathrm{p}$ 0.001]. As expected, planned comparisons revealed a significant difference between undistorted and inconsistently distorted characters $[F(1,15)=$ $10.61, \mathrm{p}<0.01]$. However, interestingly no significant difference between consistent and inconsistent distortions [F $(1,15=0.01, \mathrm{p}>0.05]$ was found. These results are illustrated in Figure 3. This pattern of results was consistent across individual characters.

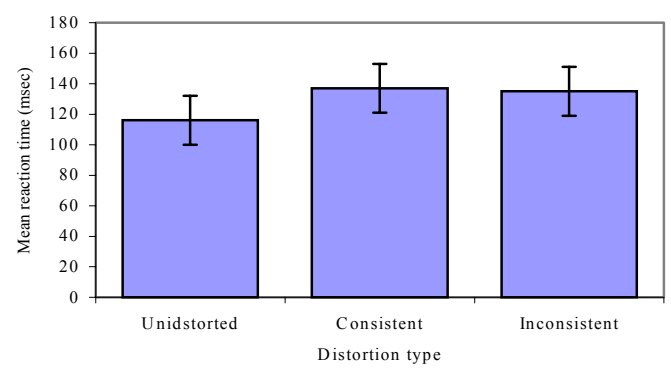

Figure 3: this figure shows the mean reaction times for each distortion-type. It can be seen that reaction times for distorted characters are similar.

Discussion The difference between consistent and inconsistent distortions was not significant. This result was not expected. A possible explanation for this finding is that the forced-choice task used during learning may not have been sensitive enough to elicit the dynamic effect. Considering that the small number of characters was well learned upon completion of the experiment, the forced-choice task used during testing may have promoted a process of discrimination as opposed to recognition.

\section{Experiment Two}

This experiment was conducted with the same motivations as Experiment One. It was felt that participants in Experiment One may have found the character recognition task easy to complete with scant attention to the complete form of the character because of the nature of the forced-choice response. In this experiment, the identification of the characters during the test phase was therefore altered to be a free choice by participants.

\section{Method}

Participants A total of 40 right-handed undergraduate students, 15 males and 25 females from the University of Hertfordshire took part in this experiment.

Materials The same Learning Set and Test Set of characters and drawing methods used in Experiment One were used here. In addition a distortion category of combined connecting and sloppy lines was added to the Test Set. This was in order to ensure that all distortion types originally used by Freyd, (1983b) were tested.

Procedure The procedure used was the same as that in Experiment One, with the exception of the test phase, in which participants were required to enter the corresponding digit for the character displayed, from memory, as opposed to the forced-choice task. This was considered to be a more powerful test than that used in Experiment One for prompting the use of dynamic information.

Results Reaction-time data recorded during testing were analysed. A two-way mixed ANOVA was conducted upon the data. Drawing method learnt (A or B) was a between-subjects factor, and type of distortion (three levels: undistorted, consistent and inconsistent) was a within subjects factor. A significant main effect of type of distortion $[\mathrm{F}(2,37)=12.782, \mathrm{p}<0.001]$ was found. As there was no main effect of drawing method $[\mathrm{F}(1,38)=0.235 \mathrm{p}>0.05]$ or interaction between method and type $[\mathrm{F}(2,76)=0.677, \mathrm{p}>0.05]$ data were collapsed across drawing methods for subsequent analysis. A further one-way ANOVA (within-subjects) was conducted upon the collapsed data. A significant effect of type of distortion was found $[F(2,38)=$ $11.515, \mathrm{p}$ 0.001]. Planned comparisons revealed a significant difference between undistorted and inconsistently distorted characters $[\mathrm{F}(1,39)=46.19, \mathrm{p}$ $<0.01]$ as expected. There was no significant difference 
between consistent and inconsistent distortions [F (1, 39 $=1.00, \mathrm{p}>0.05]$. These results are illustrated in Figure 4. This pattern of results was consistent across distortion categories and individual characters.

In addition, it is important to note that the error data logged during learning noted a number of drawing errors $^{1}$ made on certain strokes for specific characters across participants during learning. It was further observed that during recall testing, in which the drawing method used by participants to draw their characters was logged, it was noted that the majority of participants used either drawing method A or their own production processes to draw the characters.

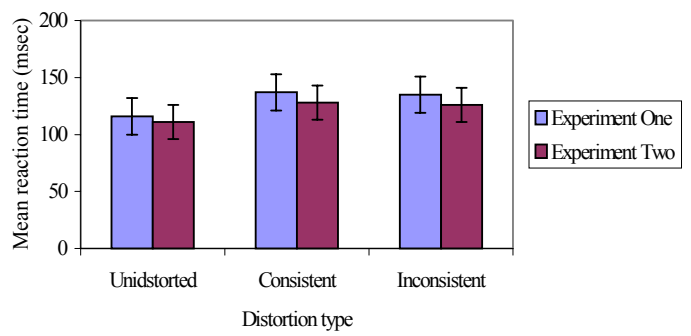

Figure 4: shows a comparison of reaction time results between Experiment One and Experiment Two. These results show a similar pattern of result across both experiments, with similar reaction times.

Discussion The results produced in this experiment concur with the findings of Experiment One, which showed no significant difference in the recognition of consistent and inconsistent distortions. The remaining explanation for the absence of the anticipated dynamic effect is that, the introduction of the motoric component generates representations during learning that interfere with the recognition process. These representations conflict with representations formed, based upon the participants' own predisposed drawing methods and create interference during the recognition process. The consequence of this is a masking of the dynamic effect. Observational data collected during learning and recall testing suggests that any character may have more than one representation.

\section{Experiment Three}

The alterations in procedure made in Experiments One and Two were done so in order to enhance the dynamic effect. However, given that these experiments did not find any evidence of such an effect, these alterations were removed and the design and methodology for the original Freyd, (1983b) experiment was revisited and followed as closely as possible. The main change in this

\footnotetext{
${ }^{1}$ An error was defined as the drawing of a stroke in a direction that deviates from the assigned drawing method.
}

experiment in comparison to the previous two experiments was that the learning phase, simply required participants to watch the characters being drawn by the computer on screen, rather than actually having the physical experience of learning to draw the characters themselves.

\section{Method}

Participants A total of 16 right-handed undergraduate students, 11 males and 5 females from the University of Hertfordshire took part in this experiment.

Materials consisted of a Learning Set of 9 undistorted characters as shown in Figure 5 which could be drawn according to two drawing methods (A or B), differing in the direction and/or sequence in which the strokes used to produce the character were drawn. The completed undistorted character was visually the same for both drawing methods. It should be noted that character three is one of the characters used in the original experiments ${ }^{2}$. There was also a Test Set consisting of 71 characters. 54 characters were distorted in a manner consistent with either drawing method A or B. The remaining 18 characters were the undistorted characters from the Learning set, repeated twice. An example of the different distortion categories used can be seen in Figure 6.

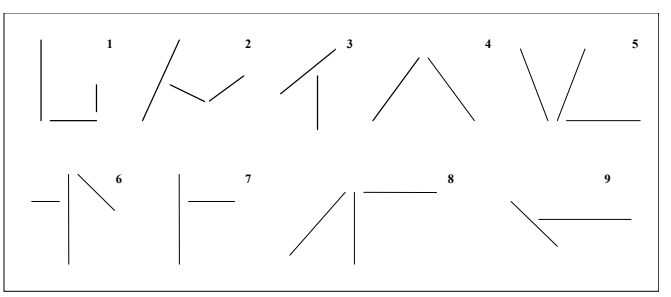

Figure 5: shows the learning set of nine undistorted characters which participants were required to draw to one of the predetermined drawing methods

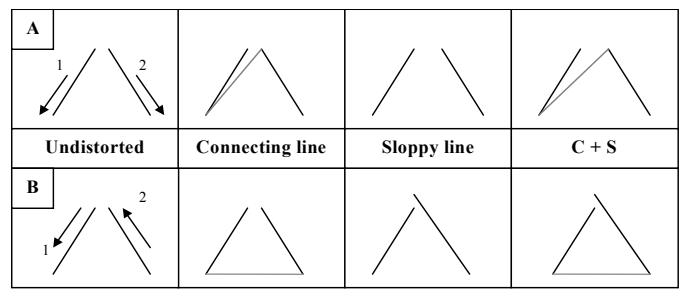

Figure 6: shows the two different drawing methods applied to a single character, and an example of a set of distortions for drawing method A (at top) and B (at bottom). Again, the distortions produced between the two drawing methods are quite different in appearance.

\footnotetext{
${ }^{2}$ There is no published complete set of the original characters in existence. All characters were designed in accordance with the requirements stated in Freyd (1983b), and with reference to the later work of Babcock and Freyd (1988).
} 
Procedure The introductory phase for this experiment was conducted in the same manner as the previous two experiments. The task for participants during the learning phase was also the same. However, participants were not given the experience of drawing the character. In place of this, participants viewed the character being drawn (according one of the selected drawing methods) and then were required to enter the corresponding digit for the character. Feedback was given as to the correctness of the response and the correct response. Participants were aware that the learning phase was complete when all characters had been identified correctly on five consecutive occasions. Following this, participants were tested using a forcedchoice task, as described in Experiment One.

Results Reaction-time data recorded during testing were analysed. A two-way mixed ANOVA conducted upon the data. Drawing method learnt (A or B) was a between-subjects factor and type of distortion (three levels: undistorted, consistent and inconsistent) was a within-subjects factor. A significant main effect of type of distortion $[\mathrm{F}(2,28)=5.466, \mathrm{p}<0.05]$ was found. As there was no main effect of drawing method $[\mathrm{F}(1,14)$ $=1.243 \mathrm{p}>0.05]$ or interaction between method and type $[\mathrm{F}(2,28)=0.237, \mathrm{p}>0.05]$ data were collapsed across drawing methods for subsequent analysis. A further one-way ANOVA (within-subjects) was conducted upon the collapsed data. A significant effect of type of distortion was found $[\mathrm{F}(2,30)=3.979, \mathrm{p}<$ 0.05]. Planned comparisons revealed a significant difference between undistorted and inconsistently distorted characters as in Freyd (1983b) $[F(1,15)=$ $4.63, \mathrm{p}<0.05]$. However, unlike the original work of Freyd, no significant difference between consistent and inconsistent distortions $[\mathrm{F}(1,15)=0.762, \mathrm{p}>0.05]$ was found. These results are shown in Figure 7 in comparison with the original results of Freyd, (1983b).

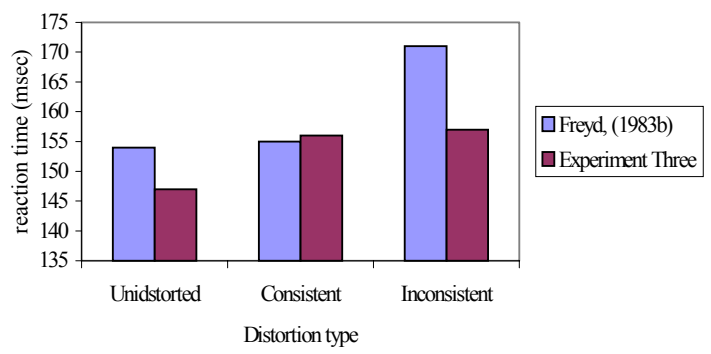

Figure 7: shows a comparison of results from Experiment Three with the original experiment conducted by Freyd, (1983b). It can be seen that between experiments, the reaction times for undistorted and consistently distorted characters are very similar.

However, the difference in findings lies with the inconsistent distortions
This pattern of results was consistent across distortion categories and for the majority of individual characters, including character two originally used in Babcock and Freyd (1988) and reported as being responsible for a very strong dynamic effect. Interestingly, although character three (an original character, with the same drawing methods as used in the original experiment) showed no significant differences between consistent and inconsistent distortions, the pattern of reaction times (consistent faster than inconsistent) was concurrent with that of Freyd (1983b).

Discussion Notably, the results of this experiment showed comparable reaction times with those of Freyd (1983b), and a significant difference between undistorted and inconsistently distorted characters. However, crucially, this pattern of results did not extend to show a significant difference between consistently and inconsistently distorted characters as in Freyd (1983b).

This is a very interesting finding and suggests that the dynamic effect is perhaps not as robust as first thought. The effect may be limited to a specific set of characters. The trend noted for character three (an original character) in comparison to other characters is supportive of this account. Surprisingly, no dynamic effect was noted for character two, originally used in Babcock and Freyd (1988) and reported as being responsible for a very strong dynamic effect. Moreover, all characters used were designed according to the requirements set by Freyd (1983b). Also, two of the characters designed for the Learning Set were done so in order to exploit the properties of diagonal-obtuse line-types and common production processes. These line-types have a relatively equal frequency of occurrence of being drawn either upwards or downwards, within the context of capital letters of the alphabet, (TR: Richardson, 2000) and were noted in Babcock and Freyd (1988) as being the stroke type for which the most pronounced dynamic effects were obtained. This was considered to be because, characters containing these stroke-types were not subject to the same level of interference from participants own production processes, as the directional drawing preference for these line-types is less strong. With these considerations in mind the possible reason for this outcome should be weighed very carefully.

\section{Conclusions}

In summary, the findings of all three experiments showed no significant difference between consistent and inconsistent distortion types. In order to understand this finding it is important to assess the possible differences in methodology between these experiments 
and the original Freyd (1983b) experiment as potential explanations.

Firstly, it should be noted that the main focus of learning for the first two experiments was the experience of drawing the characters as opposed to passively viewing character formation. During learning, participants exhibited signs of conflict between the drawing method to be followed and their own predisposition. For example, moving the cursor to a different starting point, indicating conflict between the participants' desire to draw a line in a specific direction and the predefined method. Observational data recorded during learning supports this account ${ }^{3}$. Interference during learning, may have resulted in the formation of conflicting representations, negating the dynamic effect found in Freyd's (1983b) study. Controlling for this within this paradigm would be difficult as it would require knowledge of the participants' own production processes, as well as the recruitment of participants who had different production processes, which when applied to specific characters would result in the generation of visually different distortions.

An alternative avenue of exploration would be to completely remove the visual element of viewing the characters whilst learning to draw. Within, this framework it would still be possible to generate visual images of the characters and any dynamic effect evidenced would present a strong case for a motoric contribution. However, one also must consider that this could be through necessity to complete the task, rather than any voluntarily useful contribution under normal drawing conditions.

The results for Experiment Three in comparison with those of Freyd (1983b), are both interesting and surprising. Given that the procedure followed the original description, (Freyd, 1983a; Freyd, 1983c) it seems that the only remaining explanation for this difference is the characters themselves. This suggests that the dynamic effect is perhaps not as robust as first thought and may be limited to a specific set of characters. However, as already highlighted in the discussion for Experiment Three, these characters were designed with respect to the originally stated requirements (Freyd, 1983b,c), with the same drawing methods applied to the characters. Nevertheless, a recurring theme, is the possible interference of participants' own production processes. For example, Babcock and Freyd (1988) conducted an experiment in which participants were required to draw a character according to the method used to produce it, when

\footnotetext{
${ }^{3}$ Unfortunately, it is not possible to report all of this data in full detail within the confines of this paper. The main type of data for comparison between the original work of Freyd (1983b) and this work was reaction time data, which is reported in this paper.
}

presented with a static depiction of the character. Although the results supported the conclusion that participants were able to infer the dynamic movements required to produce the character, observational and anecdotal accounts did emphasise interference between participants' own production processes and the actual drawing method, in some cases the participants own processes overriding the perceived information. The dynamic effect could be increased in magnitude by reducing this interference through careful consideration of the stroke-types and their arrangement within the context of a character, an issue addressed in the design of the materials for Experiment Three.

Finally, this work addresses some important issues concerning the practical complexity of determining the influence of action in perception, and highlights the issue that action in perception may act to interfere as well as potentially facilitate. Specifically, in terms of the use of dynamic representations, further work is required in order to determine how universal the dynamic effect is. This in turn requires further investigation into locating naturally occurring differences in production methods that would allow this to be tested.

\section{References}

Babcock, M., \& Freyd, J. (1988). Perception of dynamic information in static handwritten forms. American Journal of Psychology, 101, 111-130.

Barsalou, L. (1999). Perceptual symbol systems. Behavioural and Brain Sciences, 22 (4), 577-660.

Fadiga, L., Fogassi, L., Gallese, V., Rizzolatti, G. (2000). Visuomotor neurons: ambiguity of the discharge or 'motor' perception ?. International Journal of Psychophysiology, 35, 165-177.

Freyd, J. (1983a). The mental representation of action. Behavioural and Brain Sciences, 6, 145-146.

Freyd, J. (1983b) Representing the dynamics of a static form. Memory and Cognition, 11, 342-346.

Freyd, J. (1983c) Dynamic mental representations and apparent accelerated motion. Doctoral thesis. Stanford University.

Glenberg, A. (1997). What memory is for. Behavioural and Brain Sciences, 20, 1-55.

Prinz, W. (1997). Perception and action planning. European Journal of Cognitive Psychology, 9, 129154.

Richardson, F.M. (2000). Stroke properties of capital letters of the alphabet. Technical Report. University of Hertfordshire.

Rizzolatti, G. (1994). Nonconscious motor images. Behavioural and Brain Sciences, 17, 220.

Van den Berg, O., Vrana, S., \& Eelen, P. (1990). Letters from the heart: affective categorization of letter combinations in typists and nontypists. Journal of Experimental Psychology: Learning, Memory and Cognition, 16 (6), 1153-1161. 